\title{
In the development of wireless cognitive science
}

Recently, driven by the technology innovations and application requirements, wireless network technologies developed rapidly with high data rate and wide system bandwidth, which have already changed the way that people usually communication with each other. As different radio access technologies based wireless networks are deployed extensively in big cities and rural areas, we are facing challenges of heterogeneous network coexistence. Furthermore, radio spectrum is an essential and non-renewable resource for wireless communication, which is facing the spectrum scarcity and spectrum underutilized problems. Therefore, how to realize heterogeneous network convergence and how to improve spectrum efficiency are big issues in future wireless networks.

Cognitive wireless network (CWN) is defined as a wireless network employing technology to obtain knowledge of its operational and geographical environment, established policies and its internal state; to dynamically and autonomously adjust its operational parameters and protocols according to its obtained knowledge in order to achieve end-to-end network objectives; and to learn from the results obtained. In China, cognitive wireless network theory and technology has also been set as an important long-term research direction for future wireless network evolution.

The purpose of this special topic is to disseminate cutting-edge research results, highlight research challenges and open issues, and promote further research interests and activities in fundamental theories and key technologies in CWN. This special topic consists of nine research and review articles selected from over twenty high-quality submissions. The published articles address a variety aspect of fundamental theory problems and key technology issues in CWN. A review of cognitive radio networks aiming at intelligent and efficient development of wireless networks has been proposed which includes a brief introduction of the network architecture, cognitive abilities, autonomous decision making techniques, testbeds and standards [1]. Moreover, in order to improve the detection performance with multiple secondary users, a fully decentralized cooperative spectrum sensing scheme based on the consensus theory and unsupervised learning technology has been proposed and verified [2]. Additionally, the cognitive pilot channel technology as an efficient and accurate cognitive information delivery scheme has also been designed by using a dynamic mesh grouping scheme based on the fractal theory [3]. Furthermore, a novel concept of resource flow has been introduced for an intelligent and autonomous cognitive resource management under various types of resource [4]. Besides, other key technologies and applications have also been discussed in this special topic, such as the cognitive engine design [5], delay performance analysis [6], reconfiguration decision making [7], bio-inspired approaches and applications [8] and energy-efficient spectrum-aware clustering for cognitive radio sensor networks [9]. Therefore, this special topic will provide a comprehensive introduction to cognitive wireless network theory and technology, reduce the gap between science and engineering communities and promote innovations for future wireless network evolution.

1 Zhang P, Liu Y, Feng Z Y, et al. Intelligent and efficient development of wireless networks: A review of cognitive radio networks. Chin Sci Bull, 2012, 57: $3662-3676$

2 Wu Q H, Ding G R, Wang J L, et al. Consensus-based decentralized clustering for cooperative spectrum sensing in cognitive radio networks. Chin Sci Bull, 2012, 57: 3677-3683

3 Feng Z Y, Wei Z Q, Zhang Q X, et al. Fractal theory based dynamic mesh grouping scheme for efficient cognitive pilot channel design. Chin Sci Bull, 2012, 57: 3684-3690

4 Yang C G, Li J D, Sheng M, et al. Resource flow: Autonomous cognitive resource management framework for future networks. Chin Sci Bull, 2012, 57: 3691-3697

5 Dong X, Li Y, Wei S Q. Design and implementation of a cognitive engine functional architecture. Chin Sci Bull, 2012, 57: 3698-3704

6 Li X, Wang J, Li H S, et al. Delay performance analysis and access strategy design for a multichannel cognitive radio network. Chin Sci Bull, 2012, 57: 3705-3712

7 Zhang P, He Q, Feng Z Y, et al. Reconfiguration decision making in cognitive wireless network. Chin Sci Bull, 2012, 57: 3713-3722

8 He Z Q, Niu K, Qiu T, et al. A bio-inspired approach for cognitive radio networks. Chin Sci Bull, 2012, 57: 3723-3730

9 Zhang H Z, Zhang Z Y, Yuen C. Energy-efficient spectrum-aware clustering for cognitive radio sensor networks. Chin Sci Bull, 2012, 57: 3731-3739

Guest Editor: ZHANG Ping

Key Laboratory of Universal Wireless Communications of Ministry of Education, Beijing University of Posts and Telecommunications, Beijing 100876, China

Open Access This article is distributed under the terms of the Creative Commons Attribution License which permits any use, distribution, and reproduction in any medium, provided the original author(s) and source are credited. 